\title{
Integration of Up-conversion Nanoparticles and Ultrathin black Phosphorus for Efficient Photodynamic Theranostics under 808 nm NIR Light Irradiation
}

Ruichan Lv, ${ }^{\dagger}$ Dan Yang, ${ }^{\dagger}$ Piaoping Yang ${ }^{\dagger}, *$, Jiating Xu, ${ }^{\dagger}$ Fei He ${ }^{\dagger}$ Shili Gai ${ }^{\dagger}$ Chunxia Li, ${ }^{*}$

Yunlu Dai, ${ }^{\dagger}$ Guixin Yang, ${ }^{\dagger}$ and Jun $\operatorname{Lin}^{\dagger}{ }^{\dagger} *$

${ }^{\dagger}$ Key Laboratory of Superlight Materials and Surface Technology, Ministry of Education, College of Material Sciences and Chemical Engineering, Harbin Engineering University, Harbin, 150001, P. R. China, ${ }^{\ddagger}$ State Key Laboratory of Rare Earth Resource Utilization, Changchun Institute of Applied Chemistry, Chinese Academy of Sciences, Changchun, 130022, P. R. China

E-mail address: yangpiaoping@ @rbeu.edu.cn; jlin@ciac.ac.cn 


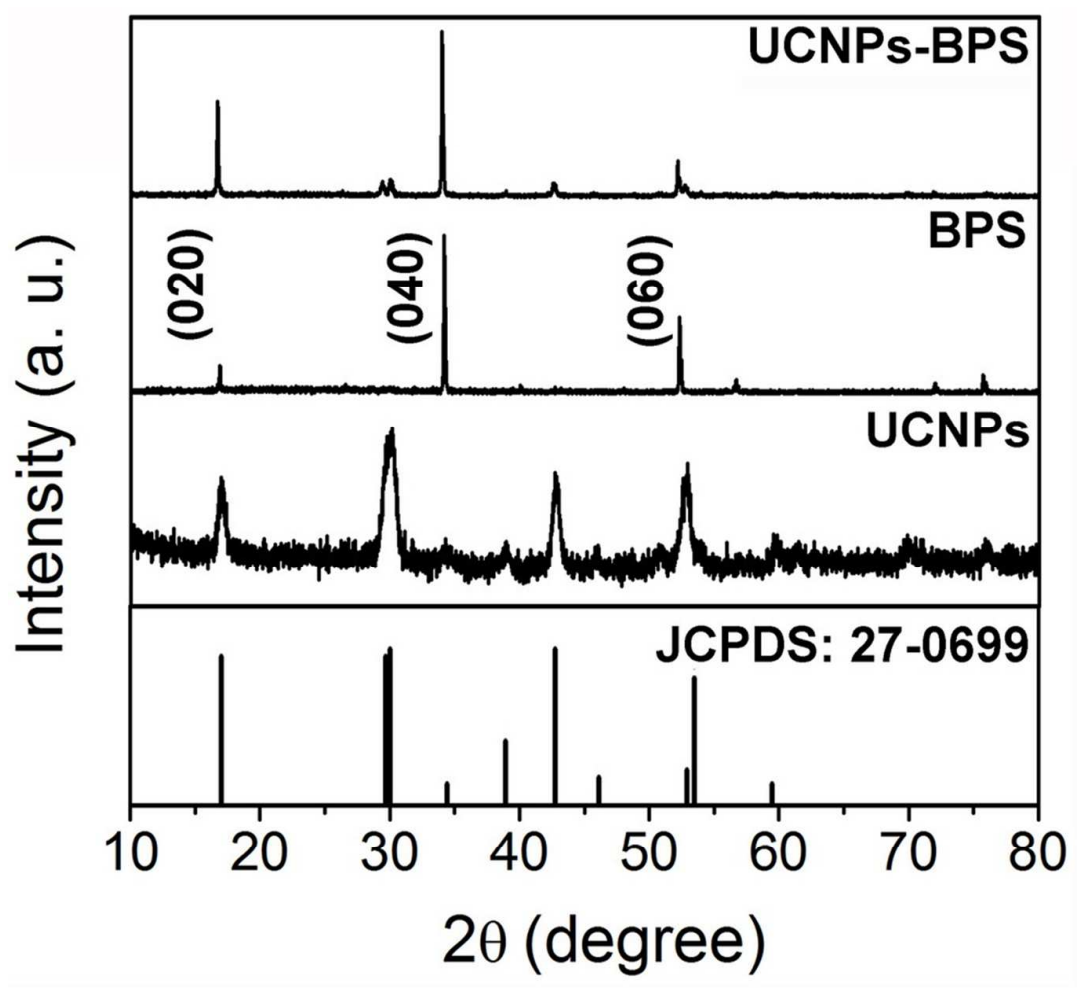

Figure S1. XRD patterns of as-synthesized samples. 

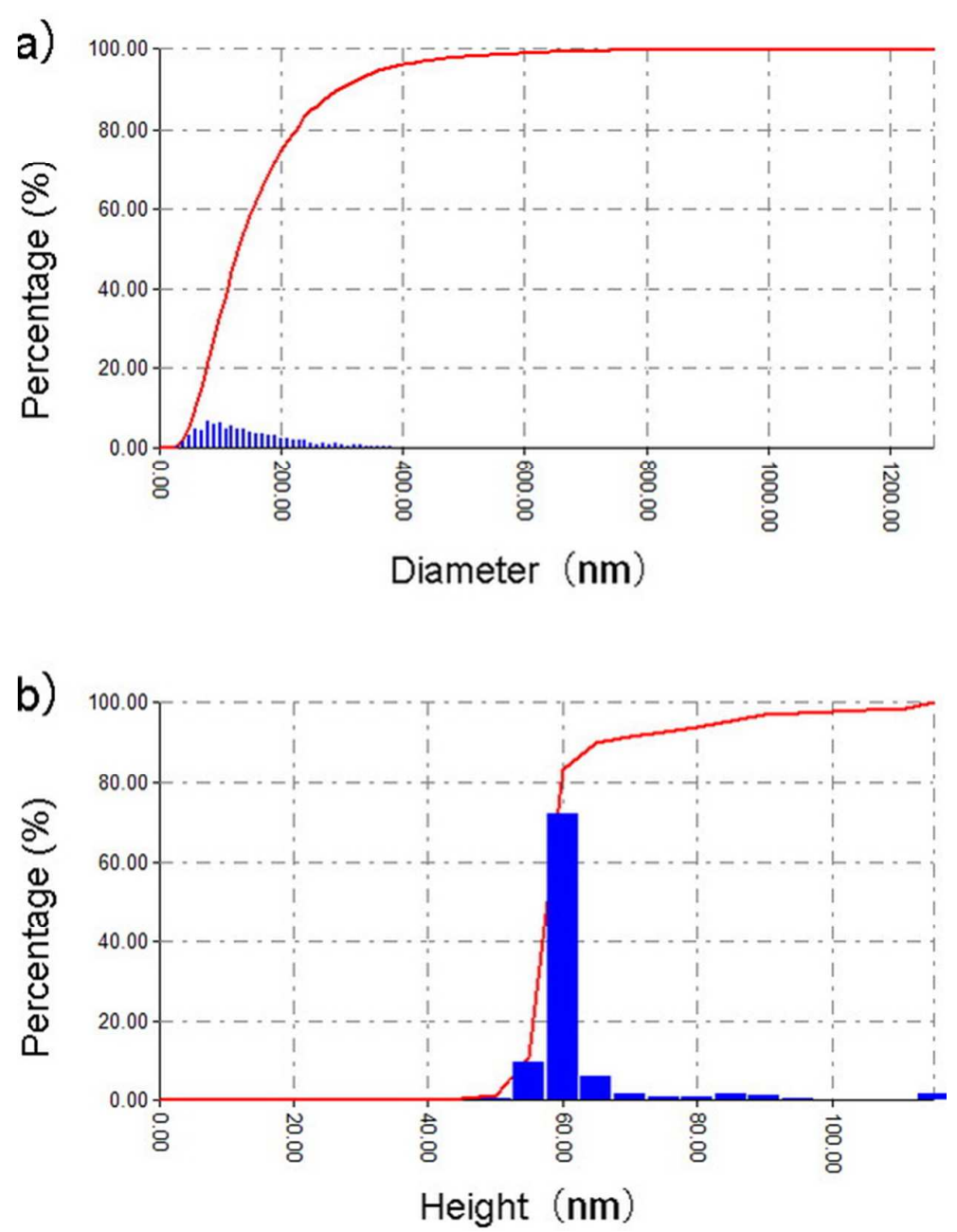

Figure S2. The statistical AFM analysis: (a) the diameter distribution and (b) the height distribution of UCNPs-BPS. 


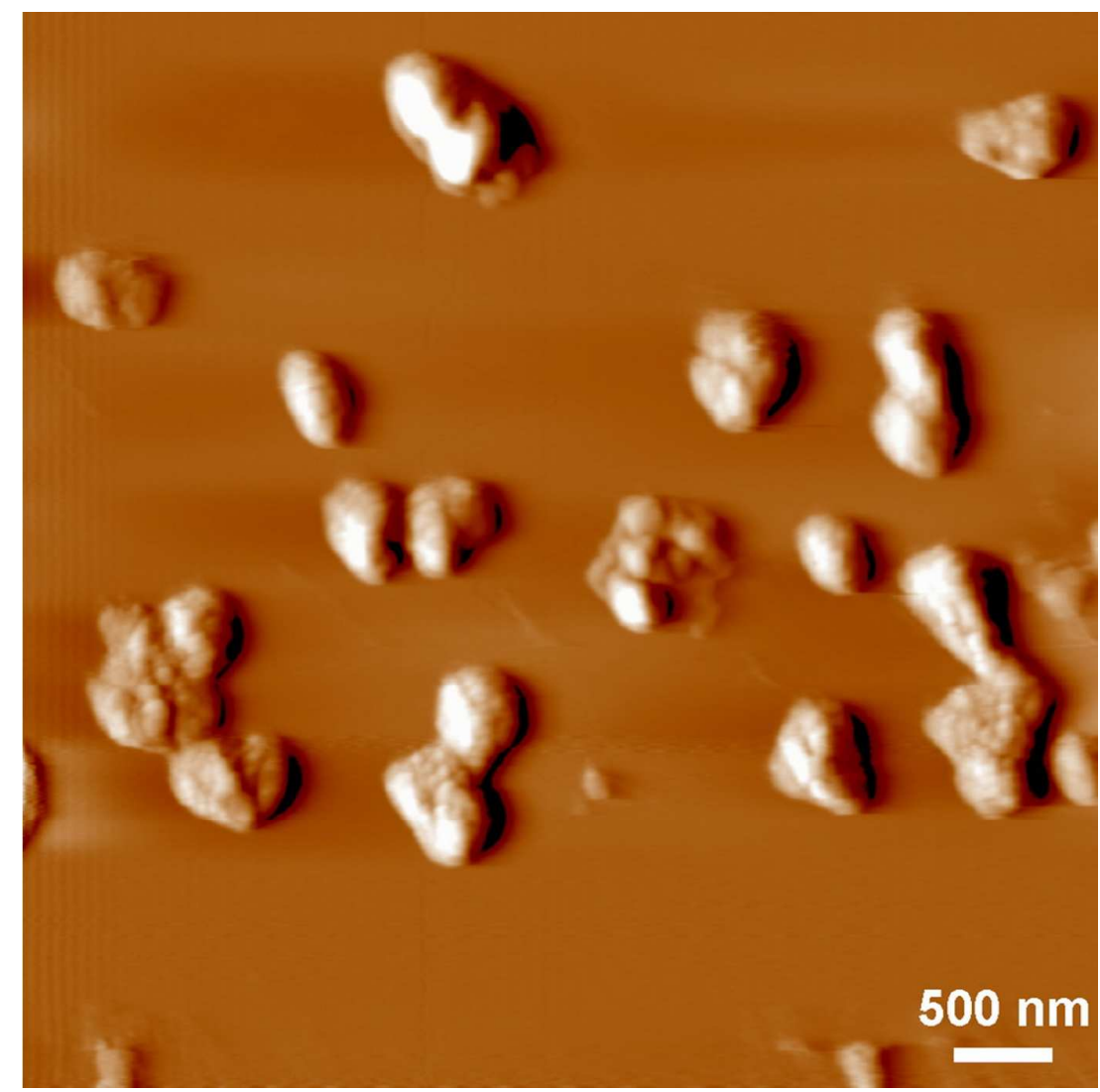

Figure S3. AFM image of UCNPs-BPS. 
a)

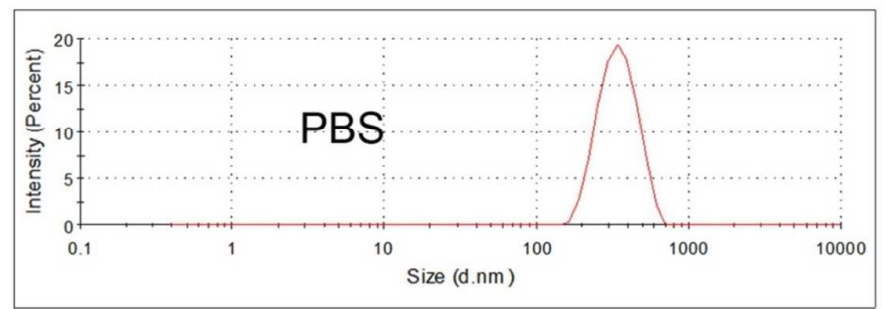

b)

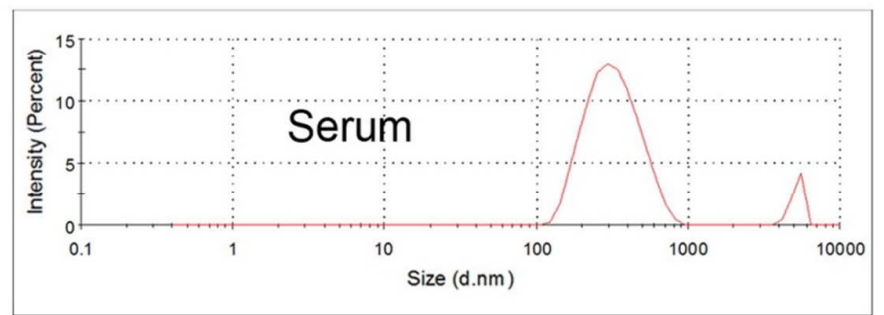

Figure S4. The dynamic light scattering properties of UCNPs-BPS dispersed in (a) PBS and (b) serum solutions. 

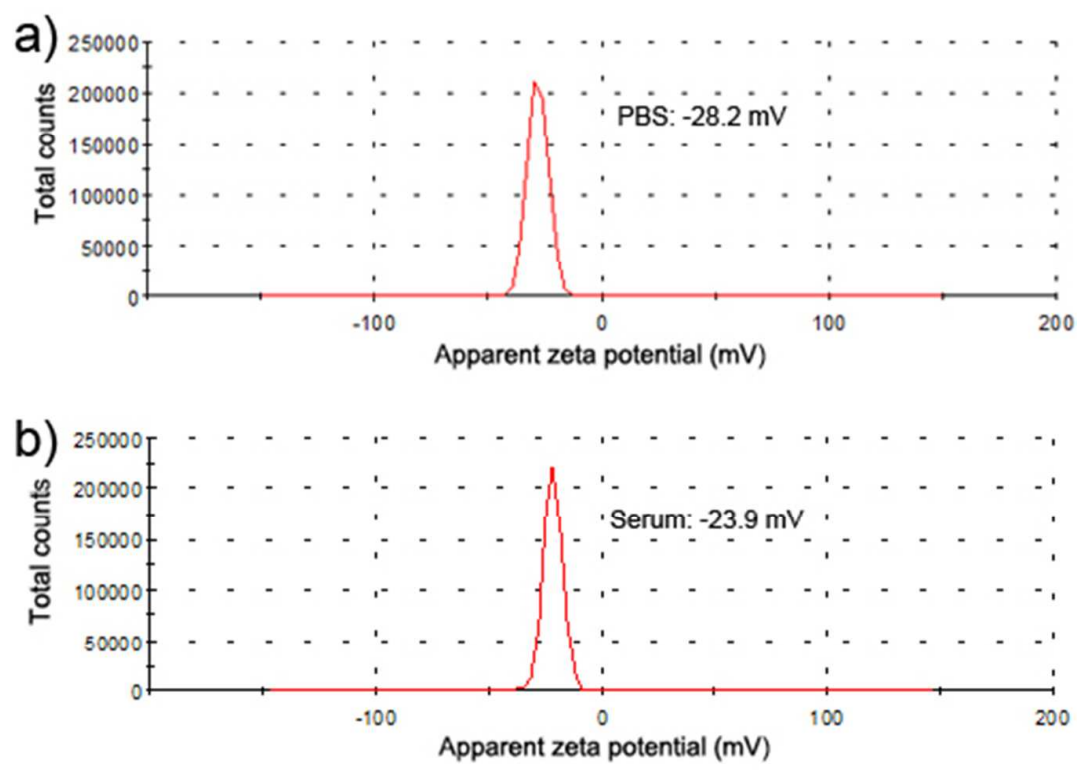

Figure S5. Zeta potential curves of UCNPs-BPS dispersed in (a) PBS and (b) serum. 


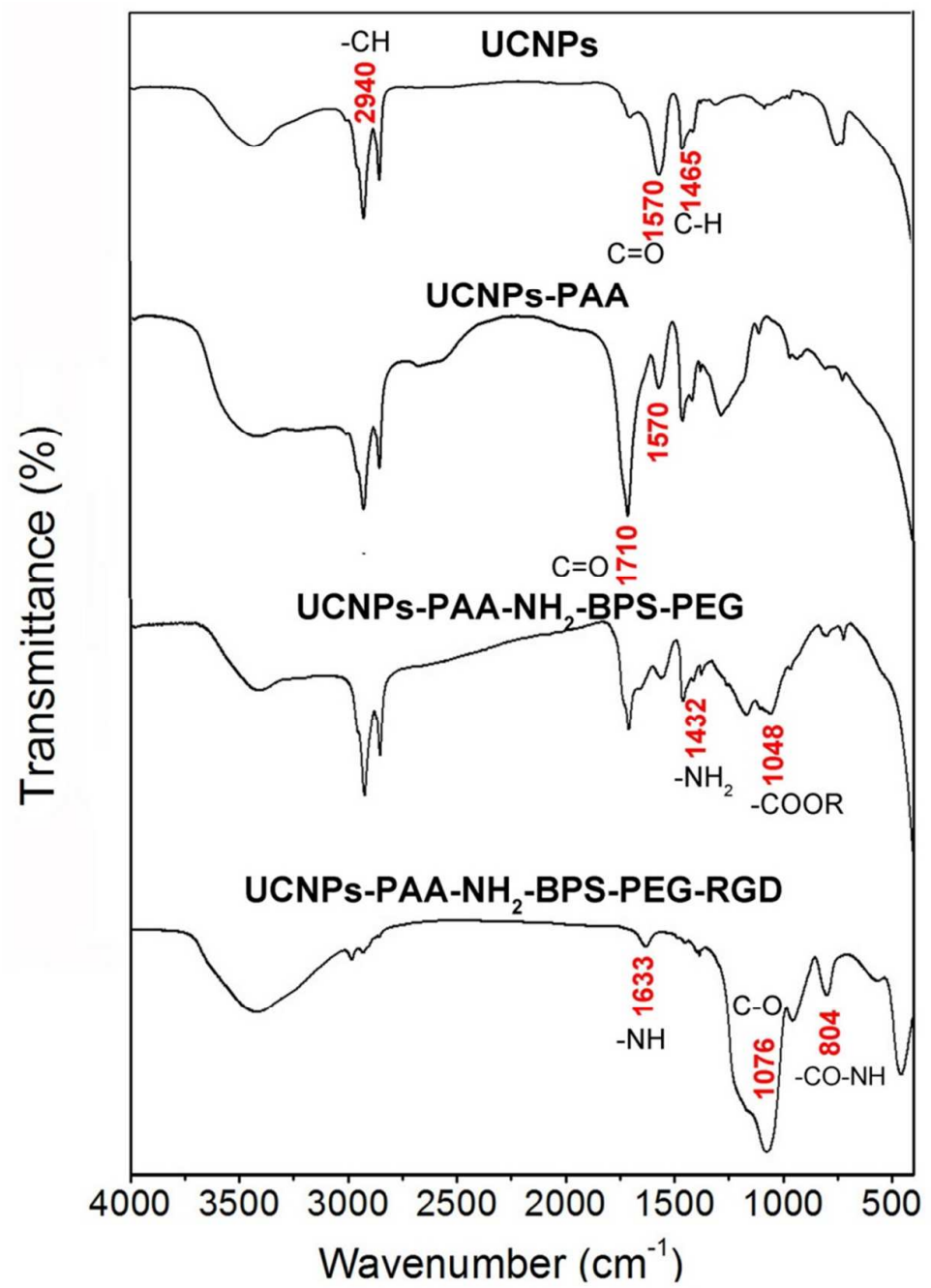

Figure S6. FT-IR spectra of the as-synthesized samples. 


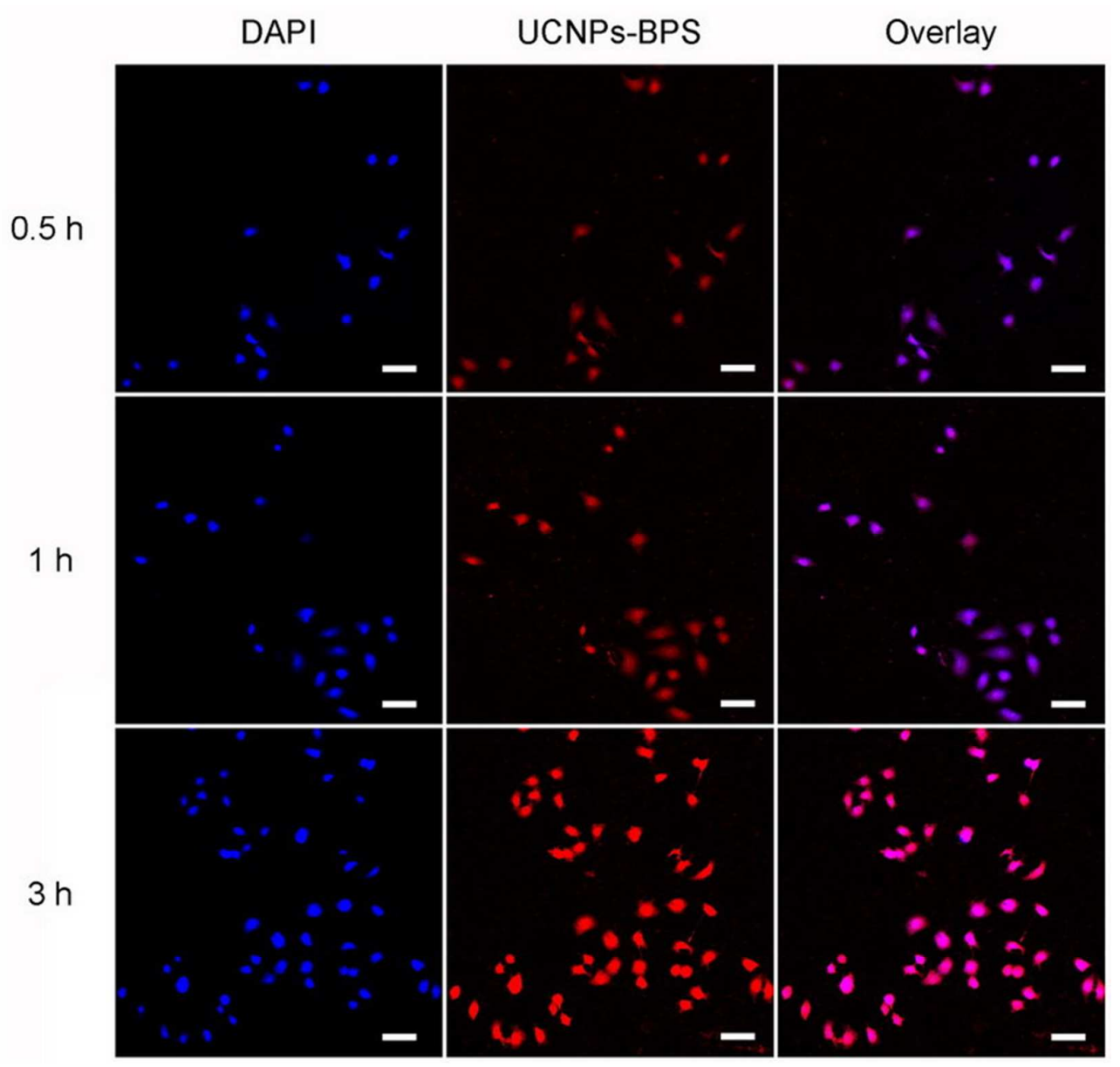

Figure S7. The confocal laser scanning microscopy images of HeLa cells with UCNPs-BPS uptaken with prolonging incubation times. All the scale bars are $50 \mu \mathrm{m}$. 


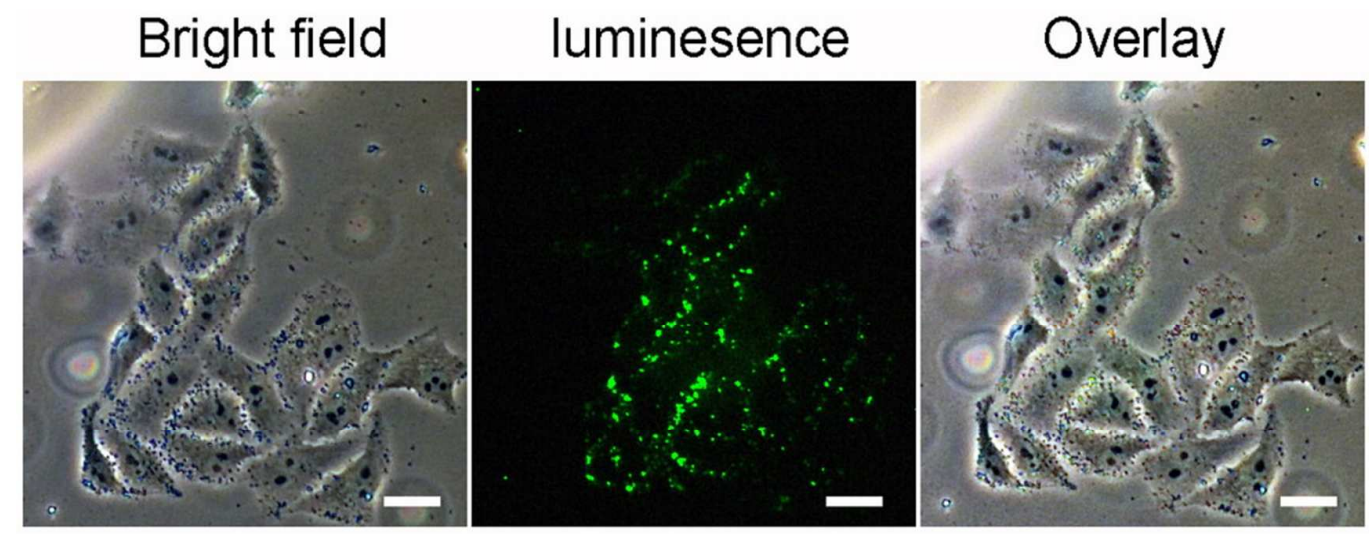

Figure S8. The inverted fluorescence microscopy images of HeLa cells incubated with UCNPs-BPS. All the scale bars are $20 \mu \mathrm{m}$ 

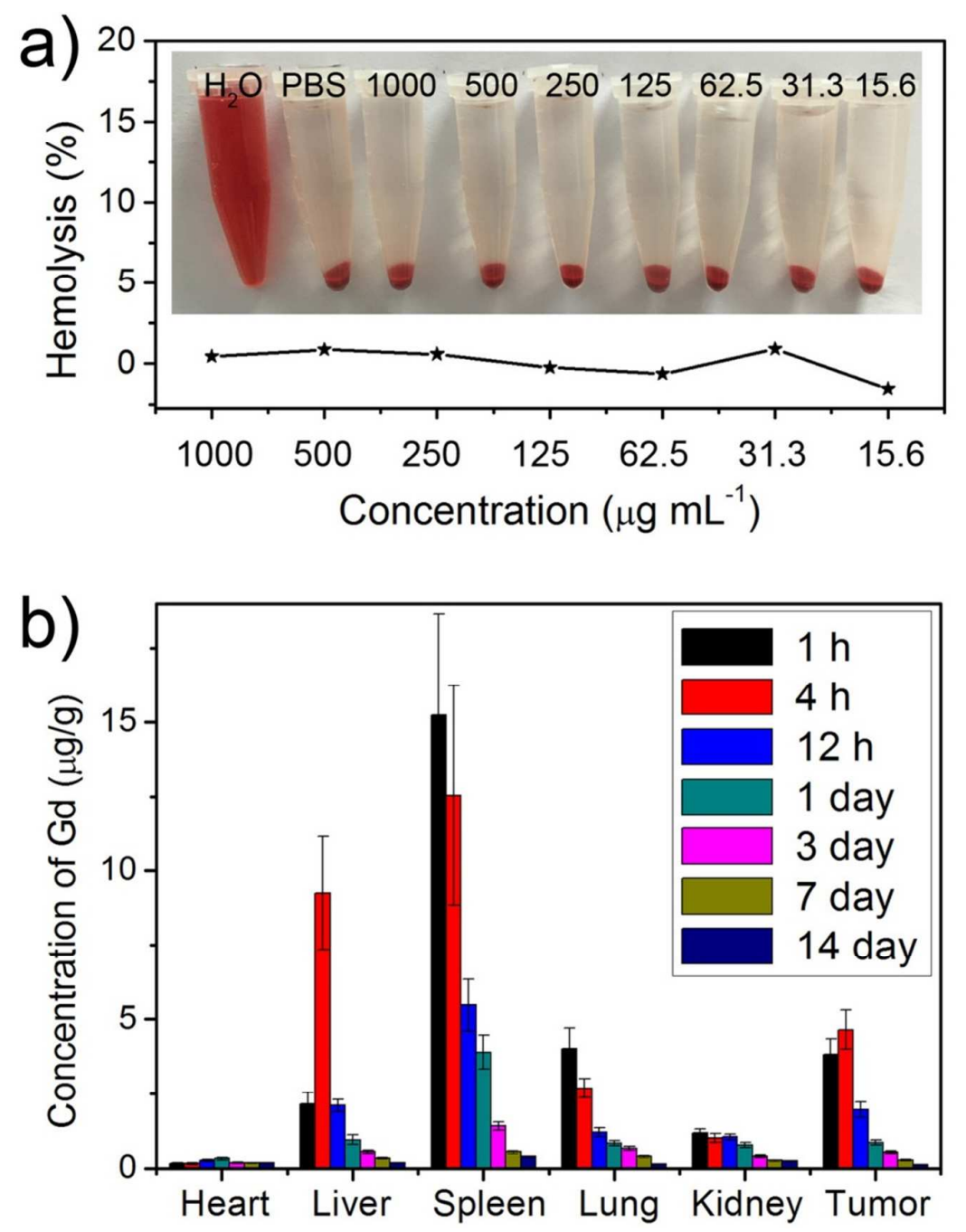

Figure S9. (a) The hemolysis property in the human blood red cells. (b) The bio-distribution in the organs and tumors after intravenous injection of UCNPs-BPS. 


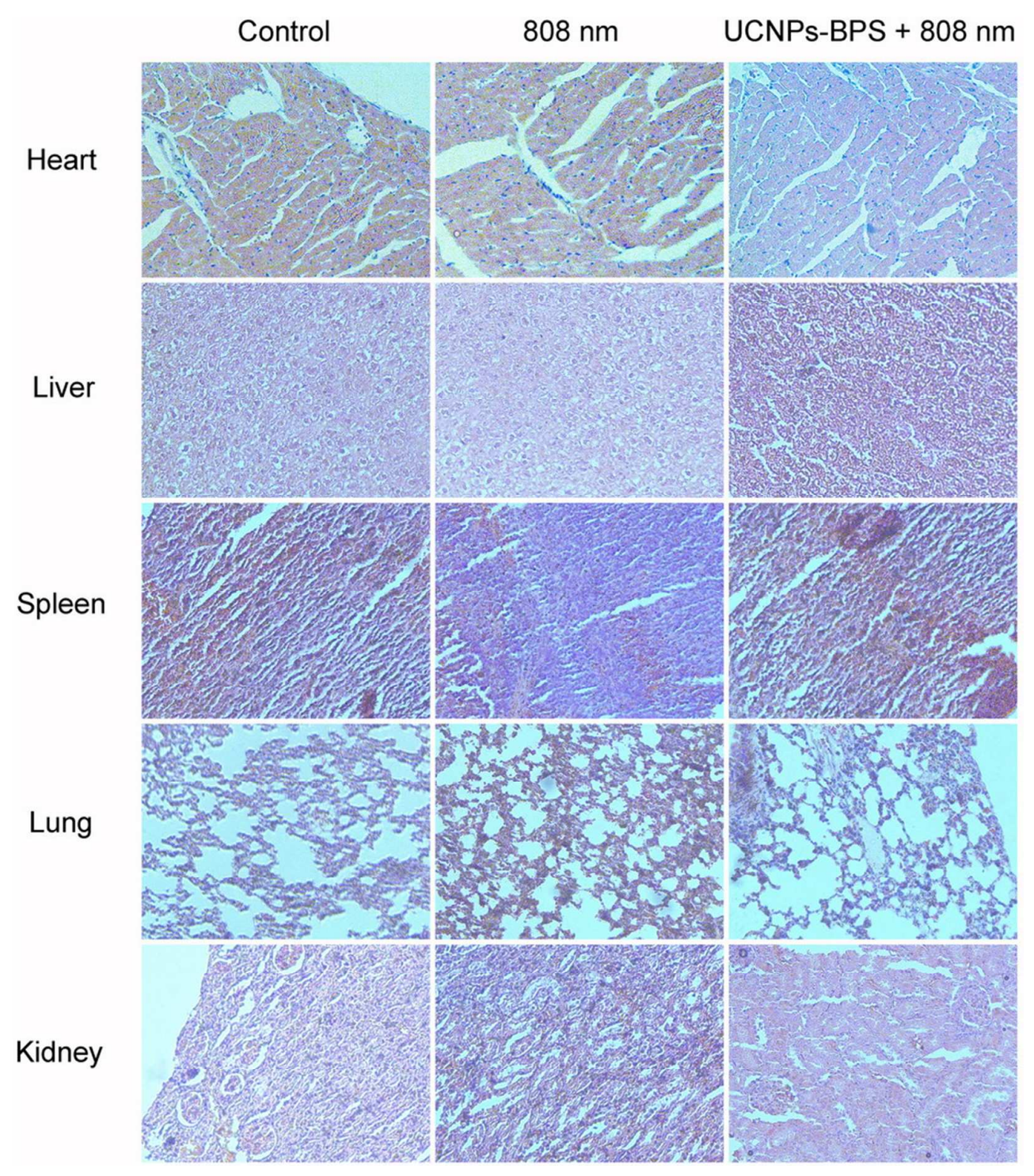

Figure S10. H\&E stained images of the tissues in the three groups. 